Research Paper

\title{
The prediction value of PI-RADS v2 score in high-grade Prostate Cancer: a multicenter retrospective study
}

\author{
Song Chen"1\#, Yun Yang2\#, Tianchen Peng1, Xi Yu³, Haiqing Deng4 and Zhongqiang Guo ${ }^{\circledR}$ \\ 1. Department of Urology, Zhongnan Hospital of Wuhan University, Wuhan, 430071, China. \\ 2. Department of Dermatology, The First Affiliated Hospital of Harbin Medical University, Harbin, 150001, China. \\ 3. Department of Urology, Renmin Hospital of Wuhan University, Wuhan, 430060, China. \\ 4. Department of Urology, Xiangyang Central Hospital, Xiangyang, 441021, China. \\ \#These authors contributed equally to this work. \\ $\square$ Corresponding author: Dr. Zhongqiang Guo, E-mail: guozhongqiang@whu.edu.cn, Tel. +86-27-6781-3104, Fax: +86-27-6781-2892.
}

(c) The author(s). This is an open access article distributed under the terms of the Creative Commons Attribution License (https://creativecommons.org/licenses/by/4.0/). See http://ivyspring.com/terms for full terms and conditions.

Received: 2020.03.06; Accepted: 2020.05.23; Published: 2020.05.30

\begin{abstract}
Background: To explore the prediction value of PI-RADS v2 in high-grade prostate cancer and establish a prediction model combined with related variables of prostate cancer.

Material and Methods: A total of 316 patients with newly discovered prostate cancer at Zhongnan Hospital of Wuhan University and Renmin Hospital of Wuhan University from December 2017 to August 2019 were enrolled in this study. The clinic information as age, tPSA, fPSA, prostate volume, Gleason score and PI-RADS v2 score have been collected. Univariate analysis was performed based on every variable to investigate the risk factors of high-grade prostate cancer. ROC curves were generated for the risk factors to distinguish the cut-off points. Logistic regression analyses were used to investigate the independent risk factors of high-grade prostate cancer. Nomogram prediction model was generated based on multivariate logistic regression analysis. The calibration curve, ROC curve, leave-one-out cross validation and independent external validation were performed to evaluate the discriminative ability, accuracy and stability of the nomogram prediction model.
\end{abstract}

Results: Of 316 patients, a total of 187 patients were diagnosed as high-grade prostate cancer. Univariate analysis showed tPSA, fPSA, prostate volume, PSAD and PI-RADS v2 score were significantly different between the high- and low-grade prostate cancer patients. Univariate and multivariate logistic regression analyses showed only tPSA, prostate volume and PI-RADS v2 score were the independent risk factors of high-grade prostate cancer. The nomogram could predict the probability of high-grade prostate cancer, with a sensitivity of $79.4 \%$ and a specificity of $\mathbf{7 7 . 6 \%}$. The calibration curve displayed good agreement of the predicted probability with the actual observed probability. AUC of the ROC curve was $0.840(0.797-0.884)$. Leave-one-out cross validation indicated the nomogram prediction model could classify $81.4 \%$ cases accurately. External data validation was performed with a sensitivity of $80.6 \%$ and a specificity of $77.3 \%$, the Kappa value was 0.5755 .

Conclusions: PI-RADS v2 score had the value in predicting high-grade prostate cancer and the nomogram prediction model may help early diagnose the high risk prostate cancer.

Key words: high-grade prostate cancer, PI-RADS v2, nomogram, prediction model

\section{Introduction}

Prostate cancer (PCa) is the second most common malignancy in male worldwide and the second leading cause of cancer death in male [1]. In recent years, the incidence and mortality of prostate tumor in China have increased year by year, and the growth rate ranks first among male malignancies [2].
PCa is usually classified as low-grade tumor and high-grade tumor. Most studies considered Gleason score (GS) $\leq 3+3$ as a low-grade PCa, GS $\geq 7$ as a highgrade $\mathrm{PCa}$, but in recent years, more and more evidence indicated that the metastasis probability, 10 -years cancer specific survival and biochemical 
recurrence after radical resection of $\mathrm{PCa}$ patients with a GS $=3+4$ were closer to patients with a GS $=3+3$ [3-5]. The treatment and prognosis of low- and highgrade prostate cancers are evidently different [6]. American Urological Association (AUA) Guideline recommended biopsy was not needed for low-grade PCa patients, only dynamic follow-up [7]. Similarly, Chinese Urological Association (CUA) Guideline also recommended dynamic monitor for PCa patients with a GS $\leq 3+4$, which could avoid erectile dysfunction, urinary incontinence and other complications after radical prostatectomy, and improve the life quality of patients; while PCa patients with a GS $\geq 4+3$ should adopt more active treatment strategies, such as radical prostatectomy, endocrine therapy or radiotherapy and chemotherapy, so as to prolong the survival time of patients [8]. It is of great significance to identify low- and high-grade prostate cancers before treatment.

At present, more and more attention is paid on multi-parameter prostate magnetic resonance imaging (MRI), which is the main method of imaging diagnosis of prostate cancer. The Prostate Imaging Reporting and Data System version 1 (PI-RADS v1) was published by European Society of Urogenital Radiology (ESUR) in 2012 and developed into the PI-RADS v2 in 2015 [9]. The PI-RADS v2 was mainly aimed at improving detection, localization, characterization, the risk stratification in patients with suspected tumor and the standardization of diagnosis and reporting of high-grade PCa [10]. Some studies indicated the PI-RADS v2 had good value in diagnosing clinically significant PCa [10-12]. Our previous study also demonstrated the predictive value of PI-RADS v2 score in PCa bone metastasis [13]. So far, several studies have confirmed age, PSA, prostate volume, PSA density (PSAD), abnormal digital rectal examination (DRE) may be the factors influencing high-grade PCa [14-17]. In addition, many prediction models have been established for highgrade PCa in foreign country, which were mainly based on PSA and derived parameters, few models incorporating multiparametric prostate MRI such as PI-RADS v2 score [18-20]. Furthermore, due to the differences in races and morbidity, it needs further confirming whether these models are appropriate for Chinese patients, and most of these studies are lack of external data validation.

Thus, we conducted a multicenter retrospective study to determine the prediction value of the PI-RADS v2 score in high-grade PCa and establish a prediction model combined with related variables of prostate cancer.

\section{Material and methods}

\section{Study patients}

The study patients consisted of a development cohort and a validation cohort. The development cohort included 316 patients with newly discovered PCa at Zhongnan Hospital of Wuhan University and Renmin Hospital of Wuhan University from December 2017 to August 2019. The clinic information as age, tPSA, fPSA, prostate volume, Gleason score and PI-RADS v2 score were collected. The pathological result of ultrasound guided prostate biopsy or radical prostatectomy was as the outcome variable. In this study, we considered GS $\leq 3+4$ as a low-grade PCa, GS $\geq 3+4$ as a high-grade PCa. We retrospectively reviewed medical records of all enrolled patients to acquire the clinical information. An independent cohort included 53 patients from Xiangyang Central Hospital (January 2018 to October 2019) was used to validate the nomogram prediction model. All patients provided the informed consent. The Ethics Committee at Zhongnan Hospital of Wuhan University had approved the using clinical information in our study (approval number: 2015029). All procedures and ethical standards were done in accordance with the national research committee and/or institutional.

\section{Inclusion criteria}

Patients were enrolled in this study if they met all the following criteria: (i) the prostate cancer patients; (ii) patients who underwent ultrasound guided prostate biopsy or radical prostatectomy; (iii) patients who underwent multiparameter MRI of the prostate (T2 WI, DWI, DCE imaging), and prostate multiparameter MRI distanced biopsy or radical prostatectomy time was within one month; (iv) had complete and detailed clinical, pathological data record.

\section{Exclusion criteria}

Patients meeting any of the following criteria were excluded: (i) merge other tumors; (ii) patients had received treatment before multiparameter MRI examination, such as hormone therapy, radiotherapy; (iii) any incomplete clinical or pathological data.

\section{Statistical analysis}

Age, fPSA and PSAD were analyzed by two-sample $t$ test and graded variables were analyzed with Mann-Whitney test or Chi-square test. Univariate analysis was performed based on every variable to investigate the risk factors of high-grade PCa. Receiver operating characteristic (ROC) curves were generated for the risk factors to distinguish the 
cut-off points and the areas under the curves (AUCs) were compared. Univariate and multivariate logistic regression analyses were used to investigate the independent risk factors of high-grade PCa. Nomogram prediction model was generated based on multivariate logistic regression analysis. The calibration curve was generated to assess the agreement of the nomogram-predicted probability with the actual observed probability. We estimated the prediction error of the nomogram prediction model using leave-one-out cross validation, the detail method information was as described by Simon et al [21]. ROC curve, leave-one-out cross validation and independent external validation were performed to evaluate the discriminative ability, accuracy and stability of the nomogram prediction model. We used SPSS 16.0 to perform all statistical analyses. Nomogram and calibration curve were generated with $R$ version 3.5.0 and a $p$ value $<0.05$ was considered statistically significant.

\section{Results}

\section{Patient characteristics and univariate analysis for prostate cancer}

The detailed clinical parameters of development cohort were displayed in Table 1, no significant difference was observed in clinical parameters between the two hospitals (all $\mathrm{p}>0.05$ ).

In the development cohort, 187 (59.2\%) of 316 patients were classified as high-grade PCa. The mean age was $73.1 \pm 8.5$ years and the median age was 73 years. The mean age of high-grade PCa patients was $73.5 \pm 8.1$ years, the median age was 74 years, and the mean age of low-grade PCa patients was $72.6 \pm 7.9$ years, with a median age of 72 years. Two-sample $t$ test showed that only fPSA and PSAD were significantly different between the high- and lowgrade prostate cancer patients $(\mathrm{p}<0.05)$. MannWhitney test and Chi-square indicated that tPSA, prostate volume and PI-RADS v2 were significantly different between the two groups $(p<0.05)$. The age and fPSA/tPSA had no statistical difference between two groups (Table 2).

Table 1. Clinical characteristics of prostate cancer patients in two hospitals

\begin{tabular}{|c|c|c|c|c|}
\hline Variables & All patients $(n=316)$ & Zhongnan Hospital (n=257) & Renmin Hospital (n=59) & $p$ value \\
\hline Age/years & & & & 0.818 \\
\hline Average/Median & $73.1 \pm 8.5 / 73$ & $73.0 \pm 8.2 / 73$ & $73.4 \pm 7.9 / 73$ & \\
\hline (Range) & $46-90$ & $46-90$ & $51-89$ & \\
\hline tPSA $(\mathrm{ng} / \mathrm{mL}), \mathrm{n}(\%)$ & & & & 0.176 \\
\hline Average/Median & $139.57 \pm 195.68 / 51.42$ & $143.93 \pm 184.56 / 52.85$ & $118.42 \pm 155.70 / 48.38$ & \\
\hline (Range) & $1.57-964.43$ & $1.57-964.43$ & $4.08-862.26$ & \\
\hline$\leq 10.0$ & $38(12.0)$ & 30 (11.6) & $8(13.6)$ & \\
\hline $10.1-20.0$ & $58(18.4)$ & $45(17.5)$ & $13(22.0)$ & \\
\hline $20.1-100.0$ & $113(35.8)$ & $94(36.6)$ & $19(32.2)$ & \\
\hline$>100.0$ & $107(33.9)$ & $88(34.2)$ & $19(32.2)$ & \\
\hline fPSA (ng/mL) & & & & 0.252 \\
\hline Average/Median & $11.51 \pm 12.16 / 9.45$ & $12.08 \pm 11.54 / 10.02$ & $9.17 \pm 8.63 / 8.15$ & \\
\hline (Range) & $0.13-69.16$ & $0.13-69.16$ & $0.21-54.33$ & \\
\hline Prostate volume $\left(\mathrm{cm}^{3}\right), \mathrm{n}(\%)$ & & & & 0.439 \\
\hline Average/Median & $46.15 \pm 30.83 / 43.98$ & $47.06 \pm 29.11 / 44.25$ & $44.38 \pm 20.42 / 40.78$ & \\
\hline (Range) & $11.93-261.52$ & $12.24-261.52$ & $11.93-196.40$ & \\
\hline$\leq 30$ & $112(35.4)$ & $88(34.2)$ & $24(40.7)$ & \\
\hline $30.1-60$ & $125(39.6)$ & $100(38.9)$ & $25(42.4)$ & \\
\hline $60.1-90$ & $52(16.5)$ & $45(17.5)$ & 7 (11.9) & \\
\hline$>90$ & $27(8.5)$ & $24(9.3)$ & $3(5.1)$ & \\
\hline PSAD (ng/mL/cm³) & & & & 0.901 \\
\hline Average/Median & $0.62 \pm 0.54 / 0.63$ & $0.63 \pm 0.51 / 0.63$ & $0.60 \pm 0.46 / 0.60$ & \\
\hline (Range) & $0.25-4.69$ & $0.25-4.69$ & $0.26-4.63$ & \\
\hline fPSA/tPSA, n (\%) & & & & 0.944 \\
\hline Average/Median & $0.12 \pm 0.09 / 0.11$ & $0.12 \pm 0.08 / 0.11$ & $0.12 \pm 0.07 / 0.12$ & \\
\hline (Range) & $0.04-0.61$ & $0.04-0.61$ & $0.06-0.57$ & \\
\hline$\leq 0.16$ & $227(71.8)$ & $182(70.8)$ & 45 (76.3) & \\
\hline$>0.16$ & $89(28.2)$ & $75(29.2)$ & $14(23.7)$ & \\
\hline Gleason score, $\mathrm{n}(\%)$ & & & & 0.385 \\
\hline$\leq 6$ & $86(27.2)$ & $67(26.0)$ & $19(32.2)$ & \\
\hline $3+4$ & $43(13.6)$ & $37(14.4)$ & $6(10.2)$ & \\
\hline $4+3$ & $50(15.8)$ & $41(16.0)$ & $9(15.3)$ & \\
\hline $8-10$ & $137(43.4)$ & $112(43.6)$ & $25(42.4)$ & \\
\hline PI-RADS v2 score, $n$ (\%) & & & & 0.797 \\
\hline $1-2$ & $10(3.2)$ & $8(3.1)$ & $2(3.4)$ & \\
\hline 3 & $71(22.5)$ & $59(23.0)$ & $12(20.3)$ & \\
\hline 4 & $124(39.2)$ & $100(38.9)$ & $24(40.7)$ & \\
\hline 5 & $111(35.1)$ & $90(35.0)$ & $21(35.6)$ & \\
\hline
\end{tabular}


Table 2. Univariate analysis for high-grade prostate cancer and low-grade prostate cancer

\begin{tabular}{|c|c|c|c|}
\hline Variables & Low-grade prostate cancer $(n=129)$ & High-grade prostate cancer $(n=187)$ & $p$ value \\
\hline Age/years & & & 0.350 \\
\hline $\begin{array}{l}\text { Average/Median } \\
\text { (Range) }\end{array}$ & $\begin{array}{l}72.6 \pm 7.9 / 72 \\
46-85\end{array}$ & $\begin{array}{l}73.5 \pm 8.1 / 74 \\
51-90\end{array}$ & \\
\hline tPSA (ng/mL), n (\%) & & & 0.029 \\
\hline $\begin{array}{l}\text { Average/Median } \\
\text { (Range) }\end{array}$ & $\begin{array}{l}87.04 \pm 112.65 / 32.70 \\
1.57-364.43\end{array}$ & $\begin{array}{l}161.19 \pm 184.36 / 68.57 \\
4.21-964.43\end{array}$ & \\
\hline$\leq 10.0$ & $23(17.8)$ & $15(8.0)$ & \\
\hline $10.1-20.0$ & $34(26.4)$ & $24(12.8)$ & \\
\hline $20.1-100.0$ & $43(33.3)$ & $70(37.4)$ & \\
\hline$>100.0$ & $29(22.5)$ & 78 (41.7) & \\
\hline fPSA $(\mathrm{ng} / \mathrm{mL})$ & & & 0.013 \\
\hline $\begin{array}{l}\text { Average/Median } \\
\text { (Range) }\end{array}$ & $\begin{array}{l}5.26 \pm 7.31 / 4.18 \\
0.13-17.16\end{array}$ & $\begin{array}{l}15.89 \pm 10.40 / 12.95 \\
1.64-69.16\end{array}$ & \\
\hline Prostate volume $\left(\mathrm{cm}^{3}\right), \mathrm{n}(\%)$ & & & 0.048 \\
\hline $\begin{array}{l}\text { Average/Median } \\
\text { (Range) }\end{array}$ & $\begin{array}{l}52.31 \pm 25.92 / 46.35 \\
18.50-261.52\end{array}$ & $\begin{array}{l}43.06 \pm 23.14 / 39.78 \\
11.93-204.83\end{array}$ & \\
\hline$\leq 30$ & $42(32.6)$ & $70(37.4)$ & \\
\hline $30.1-60$ & $49(38.0)$ & $76(40.6)$ & \\
\hline $60.1-90$ & $23(17.8)$ & $29(15.5)$ & \\
\hline$>90$ & 15 (11.6) & $12(6.4)$ & \\
\hline $\operatorname{PSAD}\left(\mathrm{ng} / \mathrm{mL} / \mathrm{cm}^{3}\right)$ & & & 0.041 \\
\hline $\begin{array}{l}\text { Average/Median } \\
\text { (Range) }\end{array}$ & $\begin{array}{l}0.57 \pm 0.48 / 0.58 \\
0.25-4.02\end{array}$ & $\begin{array}{l}0.66 \pm 0.51 / 0.67 \\
0.28-4.69\end{array}$ & \\
\hline fPSA/tPSA, n (\%) & & & 0.764 \\
\hline $\begin{array}{l}\text { Average/Median } \\
\text { (Range) }\end{array}$ & $\begin{array}{l}0.13 \pm 0.07 / 0.12 \\
0.05-0.61\end{array}$ & $\begin{array}{l}0.12 \pm 0.08 / 0.11 \\
0.04-0.60\end{array}$ & \\
\hline$\leq 0.16$ & $91(70.5)$ & $136(72.7)$ & \\
\hline$>0.16$ & $38(29.5)$ & $51(27.3)$ & \\
\hline PI-RADS v2 score, n (\%) & & & $<0.001$ \\
\hline $1-2$ & $8(6.2)$ & $2(1.1)$ & \\
\hline 3 & $43(33.3)$ & $28(15.0)$ & \\
\hline 4 & $44(34.1)$ & $80(42.8)$ & \\
\hline 5 & $34(26.4)$ & $77(41.2)$ & \\
\hline
\end{tabular}

\section{ROC curves were generated for the risk factors to distinguish the cut-off points}

To distinguish the cut-off points of high-grade PCa risk factors, ROC curves were generated and the AUCs were compared. The cut-off point of every variable was set based on the value of the maximum sum of the sensitivity and specificity on the ROC curve. Figure 1 and Table 3 showed that the AUCs were 0.631 (95\% CI: $0.515-0.798), \mathrm{p}=0.171)$ for age, 0.805 (95\% CI: $0.769-0.872, \mathrm{p}=0.028$ ) for tPSA, 0.730 (95\% CI: 0.627-0.819, $\mathrm{p}=0.042)$ for fPSA, 0.709 (95\% CI: $0.633-0.845, \mathrm{p}=0.057)$ for fPSA/tPSA, 0.616 (95\% CI: $0.526-0.780, \mathrm{p}=0.006)$ for prostate volume, $0.818(95 \%$ CI: 0.704-0.896, $\mathrm{p}=0.344)$ for PSAD, and 0.869 (95\% CI: $0.732-0.954, \mathrm{p}<0.001)$ for PI-RADS v2 score. The cut-off points of high-grade prostate cancer risk factors were as follows: age was $\geq 68$ years, tPSA was $\geq 16.47 \mathrm{ng} / \mathrm{mL}$, fPSA was $\geq 4.56 \mathrm{ng} / \mathrm{mL}$, fPSA/tPSA was $\leq 0.08$, prostate volume was $\leq 64.4 \mathrm{~cm}^{3}$, PSAD was $\geq 0.61 \mathrm{ng} / \mathrm{mL} / \mathrm{cm}^{3}$, PI-RADS v2 score was $\geq 4$. The Youden index, sensitivity and specificity of every variable were listed in Table 3.

\section{Univariate and multivariate logistic regression analyses}

Univariate logistic regression analysis showed that age was not the risk factors of high-grade PCa $(p>0.05)$, whereas tPSA, fPSA, fPSA/tPSA, prostate volume, PSAD and PI-RADS v2 score were the risk factors $(p<0.05)$. The $O R$ values were as follows: PI-RADS v2 score (OR=3.751), PSAD (OR=2.496), fPSA/tPSA $\quad(\mathrm{OR}=0.448), \quad$ tPSA $\quad(\mathrm{OR}=1.264), \quad$ fPSA $(\mathrm{OR}=1.172)$, prostate volume $(\mathrm{OR}=0.935)$. Furthermore, multivariate logistic regression analysis showed that only tPSA $(\mathrm{OR}=1.428, \mathrm{p}=0.029)$, prostate volume $(\mathrm{OR}=0.943, \mathrm{p}=0.041)$ and PI-RADS v2 score $(\mathrm{OR}=2.162, \mathrm{p}=0.002)$ were the independent risk factors of high-grade PCa (Table 4).

\section{Construction of nomogram and calibration curve to predict high-grade prostate cancer}

Based on the multivariate logistic regression analysis, tPSA, prostate volume and PI-RADS v2 score could be enrolled to generate the nomogram and calibration curve to predict high-grade PCa. Corresponding to each variable on the nomogram (Figure 2), the total score was calculated to predict the 
probability of infection in each patient. In the nomogram, the scores corresponding to the vertical line on the "score" ruler by all the variable values of the patient were found, accumulated the scores of all the variable values and found the vertical line of the "predictive ruler" on the accumulated "total score" ruler. The corresponding point was converted to the corresponding probability on the "High-grade PCa probability" scale according to the score on the predicted ruler, which was the probability of patient with high-grade PCa. The clinical information of each patient was included in the nomogram for matching analysis. The sensitivity was $79.4 \%$ and the specificity was $77.6 \%$. The calibration curve (Figure 3) displayed good agreement of the predicted probability with the actual observed probability for high-grade PCa, which indicated that the nomogram had good accuracy.
A

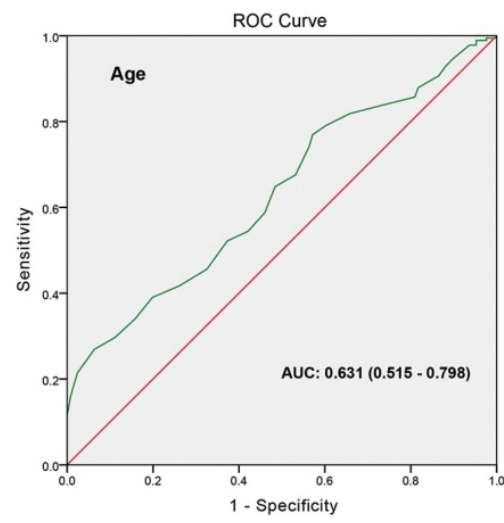

D

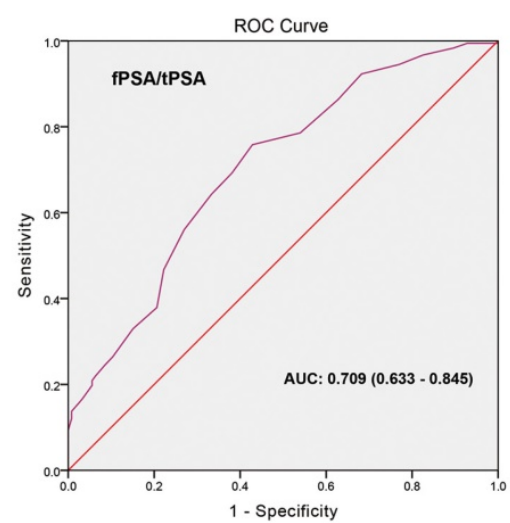

\section{G}

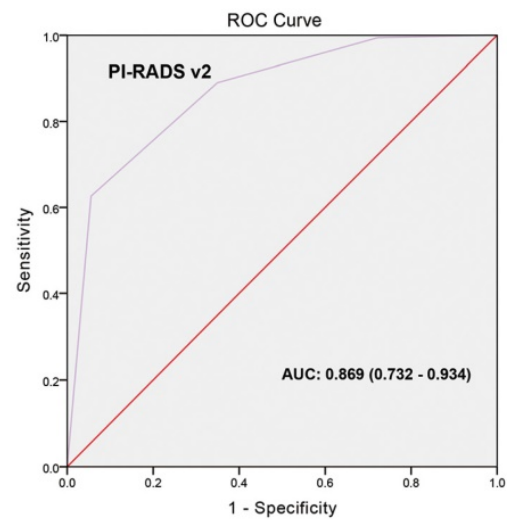

B

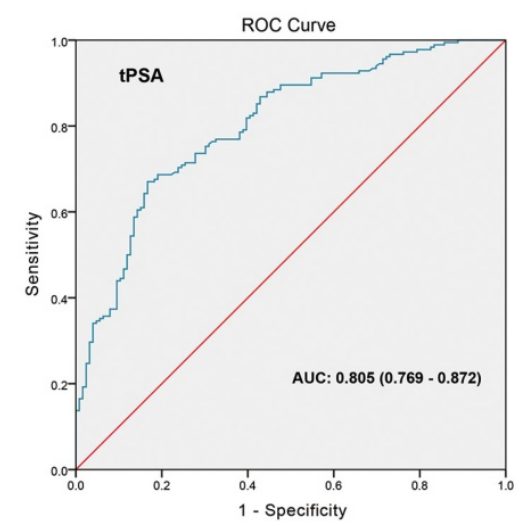

$\mathbf{E}$

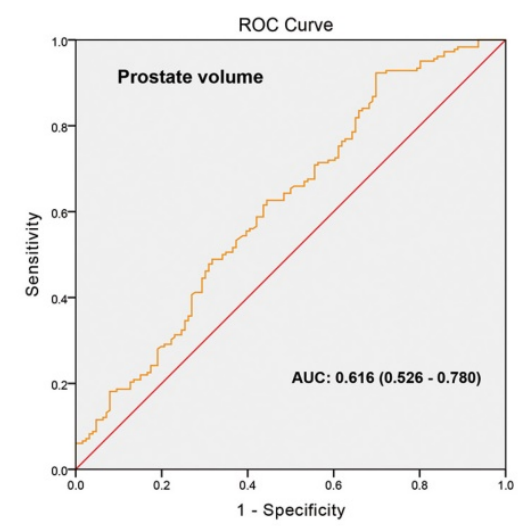

$\mathbf{F}$
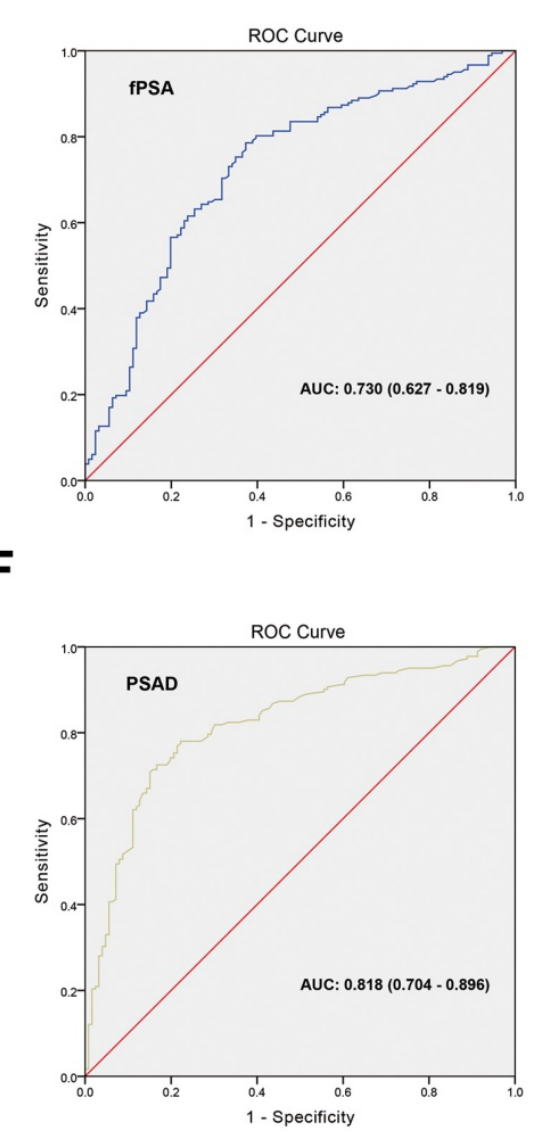

C

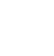


Table 3. The diagnostic value of each variable in high-grade prostate cancer

\begin{tabular}{|c|c|c|c|c|c|c|}
\hline Variables & Cut-off point & Youden index & AUC (95\% CI) & Sensitivity & Specificity & $p$ value \\
\hline Age (years) & $\geq 68$ & 0.28 & $0.631(0.515-0.798)$ & 0.77 & 0.54 & 0.171 \\
\hline tPSA $(\mathrm{ng} / \mathrm{mL})$ & $\geq 16.47$ & 0.57 & $0.805(0.769-0.872)$ & 0.81 & 0.72 & 0.028 \\
\hline fPSA (ng/mL) & $\geq 4.56$ & 0.46 & $0.730(0.627-0.819)$ & 0.61 & 0.75 & 0.042 \\
\hline fPSA/tPSA & $\leq 0.08$ & 0.43 & $0.709(0.633-0.845)$ & 0.63 & 0.71 & 0.057 \\
\hline Prostate volume $\left(\mathrm{cm}^{3}\right)$ & $\leq 64.4$ & 0.22 & $0.616(0.526-0.780)$ & 0.64 & 0.60 & 0.006 \\
\hline $\operatorname{PSAD}\left(\mathrm{ng} / \mathrm{mL} / \mathrm{cm}^{3}\right)$ & $\geq 0.61$ & 0.60 & $0.818(0.704-0.896)$ & 0.70 & 0.85 & 0.344 \\
\hline PI-RADS v2 score & $\geq 4$ & 0.67 & $0.869(0.732-0.934)$ & 0.73 & 0.88 & $<0.001$ \\
\hline
\end{tabular}

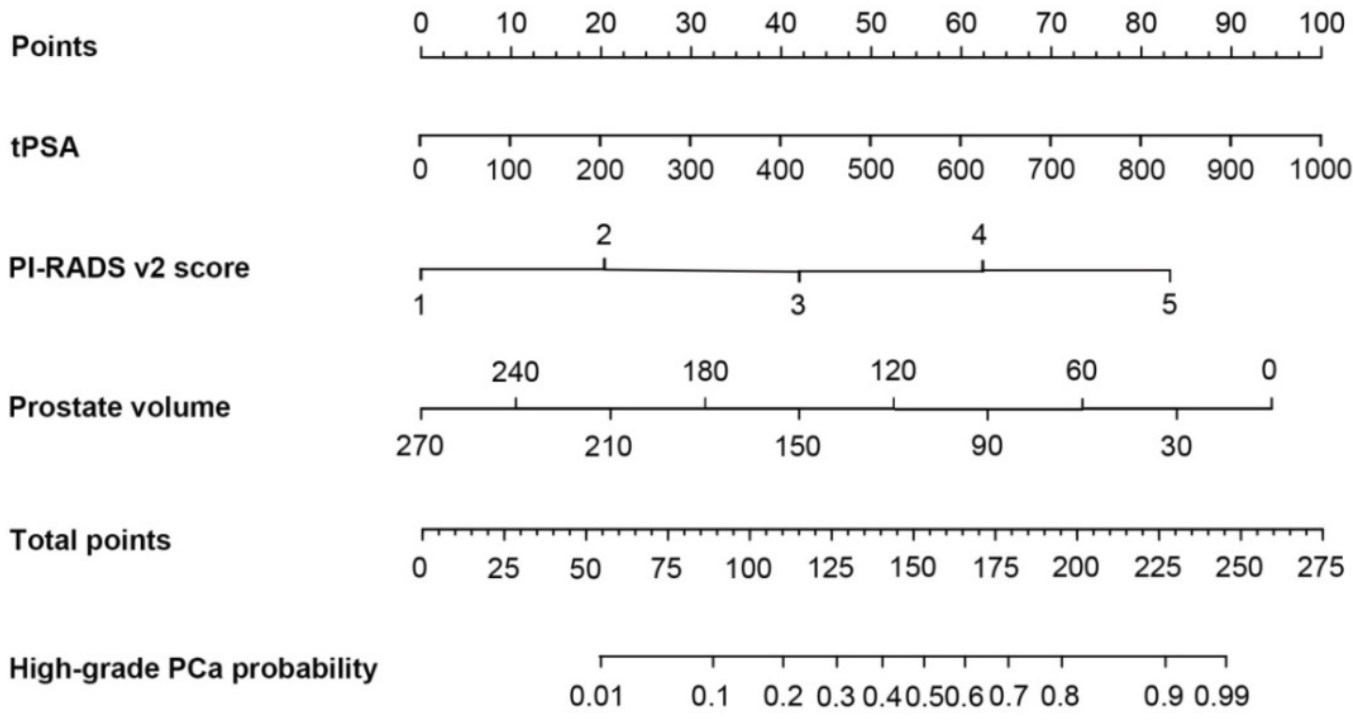

Figure 2. The nomogram was developed for high-grade prostate cancer. To estimate the risk of high-grade prostate cancer, the points for each variable were calculated by drawing a straight line from a patient's variable value to the axis labelled "Points". The score sum is converted to a probability in the lowest axis.

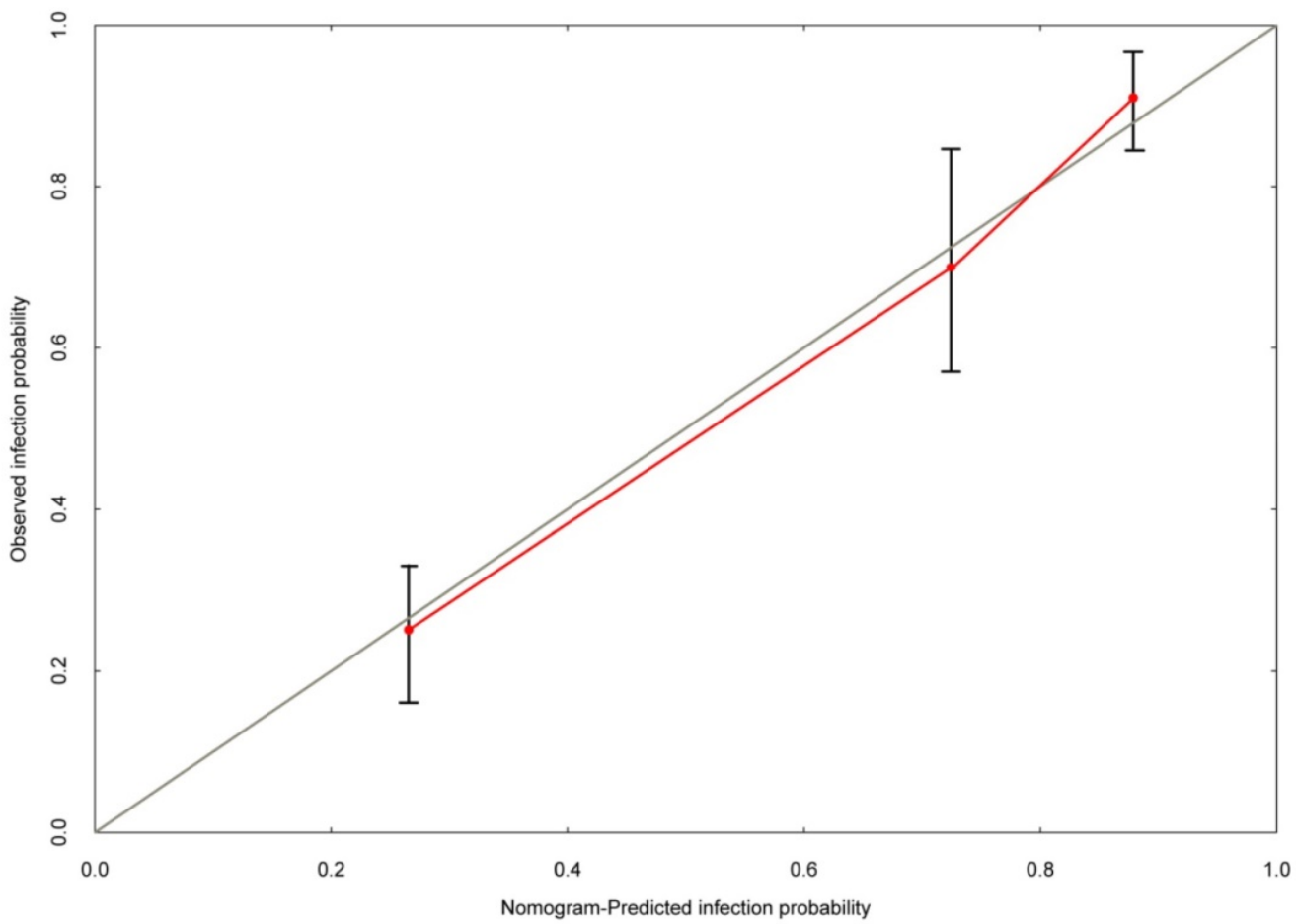

Figure 3. The calibration curve was developed for high-grade prostate cancer. The nomogram-predicted probability is plotted on the x-axis, and the actual probability is plotted on the $y$-axis. 
Table 4. Univariate and multivariate logistic regression analyses for high-grade prostate cancer

\begin{tabular}{lllllll}
\hline \multirow{2}{*}{ Variables } & \multicolumn{3}{l}{ Univariate analysis } & \multicolumn{3}{l}{ Multivariate analysis } \\
\cline { 2 - 7 } & OR & $95 \% \mathrm{CI}$ & $p$ value & OR & $95 \% \mathrm{CI}$ & $p$ value \\
\hline Age (years) & 1.033 & $0.945-1.168$ & 0.289 & 1.026 & $0.958-1.187$ & 0.536 \\
tPSA $(\mathrm{ng} / \mathrm{mL})$ & 1.264 & $1.120-1.439$ & 0.045 & 1.428 & $1.175-1.764$ & 0.029 \\
fPSA $(\mathrm{ng} / \mathrm{mL})$ & 1.172 & $1.086-1.253$ & 0.034 & 1.051 & $0.840-1.263$ & 0.718 \\
fPSA/tPSA & 0.448 & $0.253-0.826$ & 0.007 & 0.815 & $0.641-1.076$ & 0.547 \\
Prostate volume $\left(\mathrm{cm}^{3}\right)$ & 0.935 & $0.864-0.981$ & 0.023 & 0.943 & $0.912-0.990$ & 0.041 \\
PSAD $\left(\mathrm{ng} / \mathrm{mL} / \mathrm{cm}^{3}\right)$ & 2.496 & $1.787-3.642$ & 0.041 & 0.967 & $0.846-2.179$ & 0.983 \\
PI-RADS v2 score & 3.751 & $2.069-5.380$ & $<0.001$ & 2.162 & $1.473-3.548$ & 0.002 \\
\hline
\end{tabular}

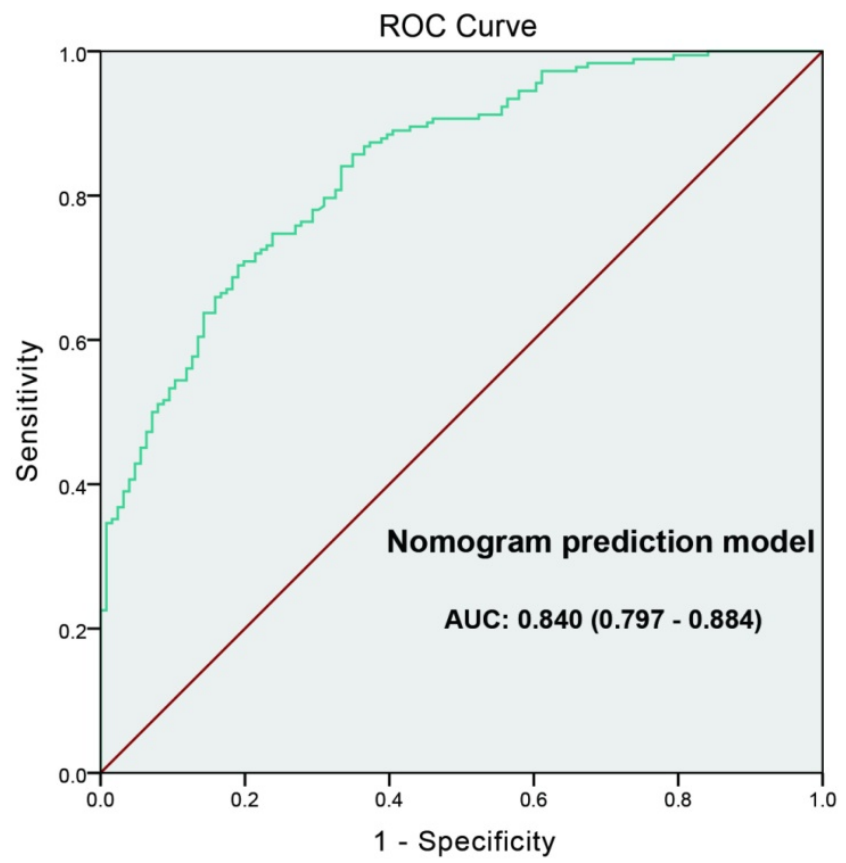

Figure 4. The ROC curve developed for nomogram prediction model of high-grade prostate cancer. The AUC of prediction model was 0.840 (0.797-0.884).

Table 5. External data validation of the prediction model for high-grade prostate cancer

\begin{tabular}{lllll}
\hline & & \multicolumn{2}{l}{$\begin{array}{l}\text { High-grade prostate cancer } \\
\text { (Validation cohort) }\end{array}$} & Total \\
\hline \multirow{2}{*}{$\begin{array}{llll}\text { High-grade prostate cancer } \\
\text { (Prediction model) }\end{array}$} & No & 17 & Yes & \\
Total & Yes & 6 & 5 & 22 \\
\hline
\end{tabular}

Sensitivity $=25 /(25+6)=80.6 \%$

Specificity $=17 /(17+5)=77.3 \%$

Kappa value $=0.5755$

\section{Evaluation of the nomogram prediction model for high-grade prostate cancer}

ROC curve was generated to evaluate the value of the nomogram prediction model; the "high-grade $\mathrm{PCa}^{\prime \prime}$ was as the outcome variable (Figure 4). The AUC of ROC curve was 0.840 (0.797-0.884). Leaveone-out cross validation indicated the nomogram prediction model could classify $81.4 \%$ cases accurately. It was been proved again that the nomogram prediction model had good discriminative ability and accuracy. To confirm the stability of the model, external data validation was performed, which was independently collected in Xiangyang Central Hospital. The sensitivity was $80.6 \%$ and the specificity was $77.3 \%$, the Kappa value was 0.5755 (Table 5).

\section{Discussion}

Pathological grade of PCa is closely related to treatment and prognosis. Some patients with highgrade PCa usually have distant metastasis, such as bone metastasis, lung metastasis. Different from non-metastatic $\mathrm{PCa}$, radical prostatectomy is no longer the main treatment. In a large sample retrospective analysis, Albertsen et al. found when the Gleason score was 8-10, patients had an obviously higher 10-year mortality rate $(12.1 \%)$ compared with low-grade PCa [22]. Therefore, it is necessary to study how to improve the diagnostic accuracy of high-grade PCa.

Most studies considered GS $\leq 3+3$ as a low-grade $\mathrm{PCa}, \mathrm{GS} \geq 7$ as a high-grade PCa, but in recent years, more and more evidence indicated that the metastasis probability, 10-years cancer specific survival and biochemical recurrence after radical resection of $\mathrm{PCa}$ patients with a $G S=3+4$ were closer to patients with a GS=3+3 [3-5]. CUA Guideline (2014 edition), European Association of Urology (EAU) Guideline (2016 edition) and European Society for Medical Oncology (ESMO) Guideline (2015 edition) stated that PCa patients with a GS $=4+3$ had remarkably different prognosis from who with a $\mathrm{GS}=3+4$, and recommended different interventions [8,23-24]. Hence, we considered GS $\leq 3+4$ as a low-grade PCa and GS $\geq 3+4$ as a high-grade PCa in this study.

The detailed clinical parameters of enrolled patients in development cohort from the two hospitals had no significant difference, demonstrating the universality of the enrolled patients. Univariate analysis showed tPSA, fPSA, prostate volume, PSAD and PI-RADS v2 were significantly different between the high-grade $\mathrm{PCa}$ patients and low-grade $\mathrm{PCa}$ patients, mainly consistent with previous studies [14-17]. We generated ROC curves and found that age, fPSA, fPSA/tPSA and prostate volume were not ideal diagnostic parameters because of low sensitivity $(<70 \%)$ or low specificity $(<70 \%)$. Park et al. demonstrated that PI-RADS v2 score could help preoperatively predict clinically significant prostate cancers, with the AUC was about 0.80 , although it was higher than AUC of tPSA, there was no statistical difference between them [10]. The results were basically consistent with our study. PSAD refers to the PSA content of a prostate per unit volume. PSAD was reported to significantly increase tumor detection rate 
and had a closely relationship with tumor invasiveness [25]. The results in our study showed that AUC of PI-RADS v2 score was the highest (0.869), and PSAD was the second (0.818). The cut-off point of PI-RADS v2 score was $\geq 4$, as same as our previous study [13]. But the cut-off point of PSAD was $\geq 0.61$ $\mathrm{ng} / \mathrm{mL} / \mathrm{cm}^{3}$, higher than $0.15-0.35$ in previous studies [15-17]. The analysis of the reasons may be as follows: (i) the scope of tPSA in this study was large (1.57-964.43 ng/ml), not limited to $4-10 \mathrm{ng} / \mathrm{ml}$; (ii) mainly for high-grade tumors, Li et al. [26] found that Gleason score $<7$ group, PSAD average \pm standard deviation was $(0.43 \pm 0.48) \mathrm{ng} / \mathrm{ml} / \mathrm{cm}^{3}$, and Gleason score $\geq 7$ group, PSAD average \pm standard deviation was $(2.55 \pm 11.06) \mathrm{ng} / \mathrm{ml} / \mathrm{cm}^{3}$, the difference $\mathrm{p}$ value between the two groups was $<0.001$. Therefore, it was reasonable to believe that when the research object was high-grade prostate cancer, the cut-off point of PSAD will increased.

In this study, univariate logistic regression analysis showed tPSA, fPSA, fPSA/tPSA, prostate volume, PSAD and PI-RADS v2 score were the risk factors $(\mathrm{p}<0.05)$. Furthermore, multivariate logistic regression analysis showed that only tPSA $(\mathrm{OR}=1.428$, $\mathrm{p}=0.029)$, prostate volume $(\mathrm{OR}=0.943, \mathrm{p}=0.041)$ and PI-RADS v2 score $(\mathrm{OR}=2.162, \mathrm{p}=0.002)$ were the independent risk factors of high-grade PCa.

Several reports in domestic and foreign have declared that nomogram had been established to predict $\mathrm{PCa}$ through prostate related parameters such as PSA [27-29]. Stamatakis et al. reported that nomogram based on magnetic resonance multiparameter imaging could be used to screen patients for dynamic follow-up, and prostate multi-parameter MRI results played an important role in clinical decision-making [30]. Tang et al. [31] established a nomogram based on PSA, prostate volume and DRE for predicting Chinese prostate cancer. The AUC of the nomogram was 0.848 , which was higher than PSA alone. Based on the age, PSA and its derived parameters, DRE and ultrasound examination results, Huang et al. [32] constructed a nomogram that reduces the number of prostate puncture biopsy needles. The AUC was 0.853, higher than PSA alone (AUC=0.761).

Based on the multivariate logistic regression analysis, we constructed nomogram and calibration curve to forecast the probability of high-grade $\mathrm{PCa}$, including tPSA, prostate volume and PI-RADS v2 score. The sensitivity was $79.4 \%$ and the specificity was $77.6 \%$. The calibration curve displayed good agreement of the predicted probability with the actual observed probability for high-grade $\mathrm{PCa}$, which indicated that the nomogram had good accuracy. In addition, ROC curve was generated to evaluate the value of the nomogram prediction model, with the AUC was 0.840 (0.797-0.884). Leave-one-out cross validation indicated the nomogram prediction model could classify $81.4 \%$ cases accurately. It was been proved again that the nomogram prediction model had good discriminative ability and accuracy. To confirm the stability of the model, external data validation was performed, which was independently collected in Xiangyang Central Hospital. The sensitivity was $80.6 \%$ and the specificity was $77.3 \%$, the kappa value was 0.5755 .

Taken together, the results showed that the nomogram prediction model exhibited good discriminative ability, accuracy and stability. It meant the prediction model had great value of prediction and could be well generalized for other independent datasets.

Unavoidable, some shortcomings in this study need to state. This study is a retrospective study, and there are inevitably biases, such as selection bias. Besides, the size of the study sample is small and it is subject to further study by expanding the sample. Moreover, although the verification of the results was relatively adequate and the cross validation, external data validation were performed verifying the discriminative ability, accuracy and stability of the nomogram, a prospective study is needed to further confirm the reliability of the results.

\section{Conclusion}

Based on 316 prostate cancer patients from two hospitals, this study evaluated the risk factors of high-grade $\mathrm{PCa}$, indicating that PI-RADS v2 score, tPSA and prostate volume were the independent risk factors of high-grade PCa. Moreover, based on the multivariate logistic regression analysis, we established a nomogram as prediction model to calculate the probability of high-grade $\mathrm{PCa}$, calibration curve, ROC curve, cross validation and external validation displayed that the nomograms had great value of prediction. The nomogram prediction model may help early diagnose the high risk prostate cancer.

\section{Abbreviations}

AUA: American Urological Association; AUCs: Areas Under the Curves; CUA: Chinese Urological Association; DRE: Digital Rectal Examination; EAU: European Association of Urology; ESMO: European Society for Medical Oncology; ESUR: European Society of Urogenital Radiology; GS: Gleason Score; MRI: Magnetic Resonance Imaging; PCa: Prostate Cancer; PI-RADS: Prostate Imaging Reporting and Data System; ROC: Receiver Operating Characteristic; PSAD: Prostate Specific Antigen Density. 


\section{Acknowledgements}

We would like to acknowledge the excellent technical assistance of Yayun Fang. This study was funded by the National Natural Science Foundation of China (grant number: 81702513) and Zhongnan Hospital of Wuhan University Science, Technology and Innovation Seed Fund Project (grant number: znpy2016018). The funders had no role in study design, data collection and analysis, decision to publish or preparation of the manuscript.

\section{Author Contributions}

S.C., Y.Y. and Z.G. conceived and designed the study, S.C., Y.Y., T.P., X.Y. and H.D. performed the analysis procedures, S.C., T.P., X.Y. and H.D. analyzed the results, S.C., T.P., X.Y. and H.D. contributed analysis tools, S.C. and Y.Y. contributed to the writing of the manuscript. All authors reviewed the manuscript.

\section{Competing Interests}

The authors have declared that no competing interest exists.

\section{References}

1. Bray F, Ferlay J, Soerjomataram I, et al. Global Cancer Statistics 2018: GLOBOCAN Estimates of Incidence and Mortality Worldwide for 36 Cancers in 185 Countries. CA Cancer J Clin. 2018; 68: 394-424.

2. Chen $W$, Zheng R, Baade PD, Zhang S, Zeng H, Bray F, et al. Cancer statistics in China, 2015. CA Cancer J Clin. 2016; 66: 115-32.

3. Starobinets O, Simko JP, Kuchinsky K, et al. Characterization and Stratification of Prostate Lesions Based on Comprehensive Multiparametric MRI Using Detailed Whole-Mount Histopathology as a Reference Standard. NMR Biomed. 2017; 30

4. Kamel MH, Khalil MI, Alobuia WM, et al. Incidence of Metastasis and Prostate-Specific Antigen Levels at Diagnosis in Gleason 3+4 Versus 4+3 Prostate Cancer. Urol Ann. 2018; 10: 203-8.

5. Wright JL, Salinas CA, Lin DW, et al. Prostate Cancer Specific Mortality and Gleason 7 Disease Differences in Prostate Cancer Outcomes Between Cases With Gleason 4+3 and Gleason 3+4 Tumors in a Population Based Cohort. J Urol. 2009; 182: 2702-7.

6. Chen RC, Rumble RB, Loblaw DA, et al. Active Surveillance for the Management of Localized Prostate Cancer (Cancer Care Ontario Guideline): American Society of Clinical Oncology Clinical Practice Guideline Endorsement. J Clin Oncol. 2016; 34: 2182-90.

7. Sanda MG, Cadeddu JA, Kirkby E, et al. Clinically Localized Prostate Cancer: AUA/ASTRO/SUO Guideline. Part I: Risk Stratification, Shared Decision Making, and Care Options. J Urol. 2018; 199 (3): 683-690.

8. Zhu X, Gou X, Zhou M. Nomograms Predict Survival Advantages of Gleason Score 3+4 Over 4+3 for Prostate Cancer: A SEER-Based Study. Front Oncol. 2019; 9: 646-54.

9. Weinreb JC, Barentsz JQ, Choyke PL, Cornud F, Haider MA, Macura KJ, et al. PI-RADS Prostate Imaging - Reporting and Data System: 2015, Version 2. Eur Urol. 2016; 69: 16-40.

10. Park SY, Jung DC, Oh YT, Cho NH, Choi YD, Rha KH, et al. Prostate Cancer: PI-RADS Version 2 Helps Preoperatively Predict Clinically Significant Cancers. Radiology. 2016; 280: 108-16.

11. Mehralivand S, Bednarova S, Shih JH, Mertan FV, Gaur S, Merino MJ, et al. Prospective Evaluation of PI-RADSTM Version 2 Using the International Society of Urological Pathology Prostate Cancer Grade Group System. J Urol. 2017; 198: 583-90.

12. Seo JW, Shin SJ, Taik Oh Y, Jung DC, Cho NH, Choi YD, et al. PI-RADS Version 2: Detection of Clinically Significant Cancer in Patients With Biopsy Gleason Score 6 Prostate Cancer. AJR Am J Roentgenol. 2017; 209: W1-W9.

13. Chen S, Wang L, Qian K, et al. Establishing a prediction model for prostate cancer bone metastasis. Int J Biol Sci. 2019; 15: 208-20.

14. Alberts AR, Roobol MJ, Verbeek JFM, et al. Prediction of High-grade Prostate Cancer Following Multiparametric Magnetic Resonance Imaging: Improving the Rotterdam European Randomized Study of Screening for Prostate Cancer Risk Calculators. Eur Urol. 2019; 75: 310-8.
15. Louie KS, Seigneurin A, Cathcart P, et al. Do prostate cancer risk models improve the predictive accuracy of PSA screening? A meta-analysis. Ann Oncol. 2015; 26: 848-64.

16. van Vugt HA, Roobol MJ, Kranse R, et al. Prediction of prostate cancer in unscreened men: external validation of a risk calculator. Eur J Cancer. 2011; 47: 903-9.

17. Gayet M, Mannaerts CK, Nieboer D, et al. Prediction of Prostate Cancer: External Validation of the ERSPC Risk Calculator in a Contemporary Dutch Clinical Cohort. Eur Urol Focus. 2018; 4: 228-34.

18. Ho T, Howard LE, Vidal AC, et al. Smoking and risk of low- and high-grade prostate cancer: results from the REDUCE study. Clin Cancer Res. 2014; 20: 5331-8.

19. Baillargeon J, Kuo YF, Fang X, et al. Long-term Exposure to Testosterone Therapy and the Risk of High Grade Prostate Cancer. J Urol. 2015; 194: 1612-6.

20. Larsen LK, Jakobsen JS, Abdul-AI A, et al. Noninvasive Detection of High Grade Prostate Cancer by DNA Methylation Analysis of Urine Cells Captured by Microfiltration. J Urol. 2018; 200: 749-57.

21. Simon R, Radmacher MD, Dobbin K, McShane LM. Pitfalls in the use of DNA microarray data for diagnostic and prognostic classification. J Natl Cancer Inst. 2003; 95 (1): 14-18.

22. Albertsen PC, Hanley JA, Fine J. 20-year outcomes following conservative management of clinically localized prostate cancer. JAMA 2005; 293: 2095-101.

23. Mottet N, Bellmunt J, Bolla M, Briers E, Cumberbatch MG, De Santis M, et al. EAU-ESTRO-SIOG Guidelines on Prostate Cancer. Part 1: Screening, Diagnosis, and Local Treatment with Curative Intent. Eur Urol. 2017; 71: 618-29.

24. Parker C, Gillessen S, Heidenreich A, Horwich A. ESMO Guidelines Committee. Cancer of the prostate: ESMO Clinical Practice Guidelines for diagnosis, treatment and follow-up. Ann Oncol. 2015; 26 Suppl 5: v69-77.

25. Verma A, St Onge J, Dhillon K, Chorneyko A. PSA density improves prediction of prostate cancer. Can J Urol. 2014; 21: 7312-21.

26. Li X, Pan Y, Huang Y, et al. Developing a model for forecasting Gleason score $\geq 7$ in potential prostate cancer patients to reduce unnecessary prostate biopsies. Int Urol Nephrol. 2016; 48: 535-40.

27. Rocco B, Sighinolfi MC, Sandri M, et al. A novel nomogram for predicting ECE of prostate cancer. BJU Int. 2018; 122: 916-8.

28. Zhu Y, Han CT, Zhang GM, et al. Development and external validation of a prostate health index-based nomogram for predicting prostate cancer. Sci Rep. 2015; 5: 15341-7.

29. Payton S. Prostate cancer: New nomogram predicts risk of Gleason upgrading. Nat Rev Urol. 2013; 10: 553

30. Stamatakis L, Siddiqui MM, Nix JW, et al. Accuracy of multiparametric magnetic resonance imaging in confirming eligibility for active surveillance for men with prostate cancer. Cancer. 2013; 119: 3359-66.

31. Tang $\mathrm{P}$, Chen $\mathrm{H}$, Uhlman $\mathrm{M}$, et al. A nomogram based on age, prostate-specific antigen level, prostate volume and digital rectal examination for predicting risk of prostate cancer. Asian J Androl. 2013; 15: 129-33.

32. Huang $Y$, Cheng G, Liu B, et al. A prostate biopsy strategy based on a new clinical nomogram reduces the number of biopsy cores required in high-risk patients. BMC Urol. 2014; 14: 8-14. 